Reprod. Nutr. Dévelop., 1988, 28 (1), 205-206.

\title{
Estimation du débit sanguin mammaire chez la vache laitière par thermodilution. 2. Résultats préliminaires
}

\author{
J. BERNABÉ, H. RULQUIN (*), J. P. CAUDAL (*), Joëlle DUVÉRÉ (*) \\ Laboratoire de Recherches sur la Traite, I.N.R.A. \\ 65, rue de Saint-Brieuc, 35042 Rennes Cedex, France. \\ (*) Station de Recherches sur la Vache Laitière, I.N.R.A. \\ Saint-Gilles, 35590 I'Hermitage, France.
}

Summary. Useful measurements of mammary blood flow (MBF) by continuous thermodilution are described in lactating cows. Vasoactive drugs increased significantly MBF (isoprenaline, oxytocin) or decreased it (adrenaline, phenylephrine). Hourly $(8.5 \%$ ) and daily $(7.7 \%)$ variations were not significant. Milking increased MBF $(+25 \%)$ and MBF was higher during the night than during the day $(P<0.05)$.

Cette note présente les conditions d'application de la technique de thermodilution continue (Rulquin et al., 1988) chez une vache vigile. Les variations journalières et horaires du débit sanguin mammaire sont estimées ainsi que les effets des traites mécaniques et l'influence d'injections i.v. de produits vaso-actifs.

Matériel et méthodes. 1) Dérivation et linéarisation chirurgicale d'une veine mammaire sous cutanée abdominale : l'isolation du vaisseau dans une gaine de peau facilite la mise en place de la sonde thermique et d'injection du soluté froid (son réchauffement par les tissus avoisinants est ainsi diminué) et limite, par section de ses collatérales, la présence de sang d'origine non mammaire (Linzell, 1966). 2) Fixation des sondes: 2 cathéters semi-rigides sont posés, sans anesthésie locale, dans la veine mammaire. Le premier (Mosquito, Vygon, $\theta=$ $2,1 \mathrm{~mm}, \mathrm{~L}=55 \mathrm{~mm}$ ) est incliné vers la mamelle et permet l'injection à contre courant dans l'axe du vaisseau. Le second (Cavafix, Bruneau, $\emptyset \mathrm{i}=1,5 \mathrm{~mm}$, Øe $=$ $2,05 \mathrm{~mm}$ ), à $10 \mathrm{~cm}$ en aval du précédent, traverse la veine mammaire de part en part. Une extrémité est obturée (mandrin) en permanence pour centrer, dans le flux sanguin, l'élément sensible du capteur de température. Pour replacer les sondes aux mêmes endroits, avec les mêmes écartement et inclinaison, nous avons conçu des manchons de fixation, légers, en silicone (Rubson), résistant aux contraintes mécaniques (lors du décubitus ventral) et limitant la contamination. Entre les mesures, le matériel est remplacé par des mandrins stériles étanches. 3) Estimation du débit sanguin : sur une vache Pie Noire (4e lactation, 30 I de lait/j) pendant 6 jours de $8 \mathrm{~h} 30$ à $17 \mathrm{~h}$ puis sur $24 \mathrm{~h}, 2$ ou 3 mesures sont effectuées toutes les $30 \mathrm{~min}$ sauf aux heures de traites où 1 essai est fait avant le massage (30 s) de la mamelle puis suivi de 3 ou 4 autres. Le $\mathrm{NaCl} 0,9 \%$ froid $\left(17 \mathrm{à} 19^{\circ} \mathrm{C}\right.$ ) est injecté rapidement (de 4,5 à $6 \mathrm{ml} / \mathrm{s}$ ). Les produits vaso-actifs sont administrés par la même voie.

Résultats et discussion. L'ensemble du système est bien toléré par l'animal (pas d'inflammation ni de traumatisme veineux, pas d'infection ni de baisse de production laitière) et l'infusion jusqu'à $12 \mathrm{l} / \mathrm{j}$ de soluté n'a entraîné ni hémodilution ni hémolyse. Grâce à cette technique nous avons pu :

- enregistrer les variations (fig. 1) attendues du débit sanguin mammaire en 
réponse à $0,3 \mathrm{mg}$ d'adrénaline $(-70 \%), 5 \mathrm{mg}$ de phényléphrine $(-55 \%)$, $0,3 \mathrm{mg}$ d'isoprénaline $(+95 \%)$ ou $15 \mathrm{Ul}$ d'ocytocine $(+25 \%)$ comme l'avait déjà montré Dhondt et al. (1973) en plus des effets sur la pression artérielle ; - mettre en évidence une répétabilité des mesures comparable à celle observée chez la chèvre par Burvenich (1980) : coefficients de variation résiduelle intraheure de $8,5 \%$ (non significatif $=$ NS), inter-heures de $9 \%$ (significatif à $5 \%=$ S), inter-jours de $7,7 \%$ (NS) et $8,5 \%$ (S) respectivement selon que les périodes de traites sont incluses ou non. Le débit mammaire baisse après la traite de $8 \mathrm{~h} 30$ (jusqu'à un minimum situé vers $12 \mathrm{~h}$ ) puis remonte avant $16 \mathrm{~h} 30$;

- observer un débit sanguin plus élevé (S) la nuit $(5,23 \mathrm{l} / \mathrm{min})$ que le jour $(4,42 \mathrm{l} / \mathrm{min})$, le maximum étant à minuit (fig. 2) (observation unique à confirmer) ; - constater, 1 minute après la pose des gobelets trayeurs, une hausse (S) du débit sanguin $(+15 \%$ le matin, $+35 \%$ le soir) qui se maintient pendant la traite.

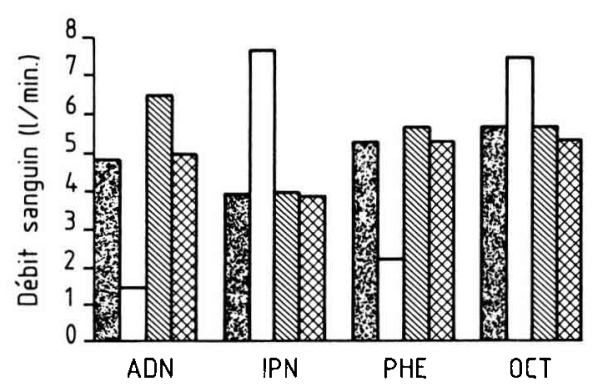

FIG. 1. - Variation du débit sanguin mammaire (1 $\mathrm{min}$ ) après une injection intraveineuse d'agonistes adrénergiques: adrénaline (ADN), isoprénaline (IPN), phényléphrine (PHE) et d'ocytocine (OCT).

$\square$ : avant injection: $\square: 60 \mathrm{~s}$ après l'injection ; QZZ: $5 \mathrm{~min}$ après l'injection : E::-3:10 min après l'injection.

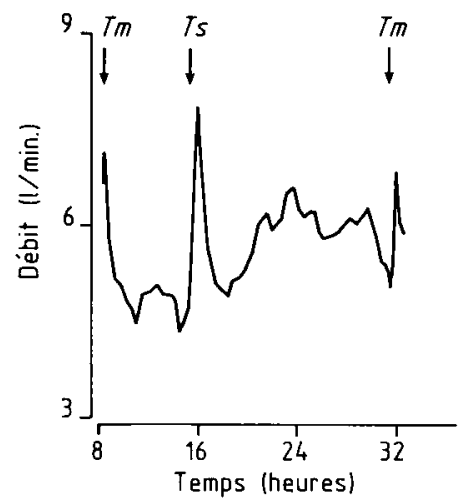

FIG. 2. - Evolution du débit sanguin dans la veine mammaire au cours du temps. Les flèches indiquent le début des traites du matin $(T \mathrm{~m})$ et du soir (Ts).

Ces premiers résultats sont encourageants et comparables à ceux obtenus par la méthode électromagnétique qui fournit des valeurs instantanées mais ne peut être employée que pendant un temps limité. Actuellement, nous confirmons la validité et la représentativité de notre méthode sur un nombre plus important d'animaux, sans ignorer que le débit dans la veine mammaire peut être inférieur au débit artériel dans l'artère inguinale; tout le sang efférent n'emprunte pas cette seule voie même si, selon Linzell (1974), le rôle des veines périnéale et pudique est minime chez la vache âgée en position debout (reflux sanguin résultant de la faible efficacité des valvules).

Burvenich C., 1980. Z. Tierphysiol., 43, 18-23.

Dhondt G., Houvenaghel A., Peeters G., Verschooten F., 1973. Arch. int. Pharmacodyn., 204, 89104.

Linzell J. L., 1966. Circulat. Res., 18, 745-754.

Linzell J. L., 1974. In Lactation : a comprehensive treatise. Vol. 1, ed. B. L. Larson, V. R. Smith. Academic Press. INC., 143-225.

Reynolds M., J. L. Linzell, F. Rasmussen, 1968. Amer. J. Physiol., 214, 1415-1424.

Rulquin H., Bernabé J., Caudal J. P., Duvéré J., 1988. Reprod. Nutr. Dévelop., 28, $203-204$. 